Article

\title{
Approximate Controllability of Infinite-Dimensional Degenerate Fractional Order Systems in the Sectorial Case
}

\author{
Dumitru Baleanu ${ }^{1,2}$, Vladimir E. Fedorov ${ }^{3,4, * \mathbb{C}}$, Dmitriy M. Gordievskikh ${ }^{5}$ and Kenan Taş ${ }^{1}$ \\ 1 Department of Mathematics, Faculty of Arts and Sciences, Çankaya University, TR-06530 Ankara, Turkey \\ 2 Institute of Space Science, R-077125 Măgurle-Bucharest, Romania \\ 3 Department of Mathematical Analysis, Chelyabinsk State University, 454001 Chelyabinsk, Russia \\ 4 Laboratory of Functional Materials, South Ural State University, 454080 Chelaybinsk, Russia \\ 5 Department of Physics, Mathematics and Information Technology Education, \\ Shadrinsk State Pedagogical University, 641870 Shadrinsk, Russia \\ * Correspondence: kar@csu.ru; Tel.: +7-952-514-1719
}

Received: 1 June 2019; Accepted: 9 August 2019; Published: 12 August 2019

\begin{abstract}
We consider a class of linear inhomogeneous equations in a Banach space not solvable with respect to the fractional Caputo derivative. Such equations are called degenerate. We study the case of the existence of a resolving operators family for the respective homogeneous equation, which is an analytic in a sector. The existence of a unique solution of the Cauchy problem and of the Showalter-Sidorov problem to the inhomogeneous degenerate equation is proved. We also derive the form of the solution. The approximate controllability of infinite-dimensional control systems, described by the equations of the considered class, is researched. An approximate controllability criterion for the degenerate fractional order control system is obtained. The criterion is illustrated by the application to a system, which is described by an initial-boundary value problem for a partial differential equation, not solvable with respect to the time-fractional derivative. As a corollary of general results, an approximate controllability criterion is obtained for the degenerate fractional order control system with a finite-dimensional input.
\end{abstract}

Keywords: approximate controllability; degenerate evolution equation; fractional Caputo derivative; sectorial operator

MSC: 93B05; 35R11; 34G99

\section{Introduction}

Infinite-dimensional systems with distributed control, whose dynamics are described by the fractional order equation of the form

$$
D_{t}^{\alpha} L x(t)=M x(t)+f(t), \quad t \in(0, T],
$$

are studied. Here $\mathcal{X}$ and $\mathcal{Y}$ are reflexive Banach spaces, $L, M: \mathcal{X} \rightarrow \mathcal{Y}$ are linear closed operators, defined on dense in $\mathcal{X}$ linear subspaces $\mathcal{D}_{L}$ and $\mathcal{D}_{M}$ respectively, $m-1<\alpha \leq m \in \mathbb{N}, D_{t}^{\alpha}$ is the Caputo derivative, $f \in C^{\gamma}([0, T] ; \mathcal{Y}), \gamma \in(0,1]$, where $C^{\gamma}([0, T] ; \mathcal{Y})$ is the space of Hölder functions (see the definition before Theorem 1). Equation (1) is supposed to be degenerate, that is, $\operatorname{ker} L \neq\{0\}$, and the pair $(L, M)$ generates an analytic in a sector resolving operators family of the 
homogeneous $(f \equiv 0)$ Equation (1). The existence of a unique solution of the Cauchy problem and of the Showalter-Sidorov problem

$$
(L x)^{(k)}(0)=y_{k} \in \mathcal{Y}, \quad k=0,1, \ldots, m-1,
$$

to the inhomogeneous degenerate Equation (1) is proved and the form of the solution is also derived.

The approximate controllability is investigated for distributed systems of control of the form

$$
D_{t}^{\alpha} L x(t)=M x(t)+B(t) u(t)+g(t), \quad t \in(0, T],
$$

with $g \in C^{\gamma}([0, T] ; \mathcal{Y}), \gamma \in(0,1], B \in C^{\gamma}([0, T] ; \mathcal{L}(\mathcal{U} ; \mathcal{Y}))$, where $\mathcal{U}$ is a Banach space, $u \in C^{\gamma}([0, T] ; \mathcal{U})$ is a control function. Taking into account the obtained results on the initial problems to the degenerate equation, the initial state is determined by the Showalter-Sidorov conditions, not by the Cauchy conditions. The equivalence of the approximate controllability of the original degenerate system and of two its subsystems on the degeneration subspace and its complement is proved. Based on this result, the obtained criteria of the approximate controllability of the subsystems are used to get a criterion for the whole degenerate control system. The criterion is illustrated by the application to an initial-boundary value problem for a partial differential equation with a degenerate spatial differential operator at the Caputo time derivative. As a corollary of the general result, an approximate controllability criterion is obtained for the degenerate fractional order control system (3) with a finite-dimensional input, that is, when $\mathcal{U}=\mathbb{R}^{n}$.

In the case of $\mathcal{X}=\mathcal{Y}, L=I, \alpha=1$ controllability and approximate controllability issues have been studied in classical papers [1-5], and in many other works (see the surveys in References [6,7]). For fractional $\alpha$ see References [8,9] and others.

For various classes of degenerate (ker $L \neq\{0\})$ systems (3) of the order $\alpha=1$ the controllability and the approximate controllability were researched in References [10-14]. In References [15-17] the approximate controllability issues are studied for system (3) of fractional order $\alpha$ under the condition of $(L, p)$-boundedness of the operator $M$, it is a more restrictive condition on the pair of operators $L$, $M$ than in this work.

The solvability of various optimal control problems for systems, described by Equation (3) with $(L, p)$-bounded operator $M$ and respective semilinear equations, is studied in References $[18,19]$ and others.

\section{Nondegenerate System Solvability}

To study the approximate controllability of fractional order control systems, we formulate the existence and uniqueness theorems for the equations, which describe their dynamics. Firstly, we consider the equation, which is resolved with respect to the fractional derivative.

Denote $g_{\beta}(t)=t^{\beta-1} / \Gamma(\beta)$ at $t>0, \beta>0$, where $\Gamma(\cdot)$ is the Euler Gamma function,

$$
J_{t}^{\beta} h(t):=\left(g_{\beta} * h\right)(t):=\frac{1}{\Gamma(\beta)} \int_{0}^{t}(t-s)^{\beta-1} h(s) d s .
$$

Let $m-1<\alpha \leq m \in \mathbb{N}, D_{t}^{\alpha}$ is the fractional Caputo derivative, that is,

$$
D_{t}^{\alpha} h(t):=D_{t}^{m} J_{t}^{m-\alpha}\left(h(t)-\sum_{k=0}^{m-1} h^{(k)}(0) g_{k+1}(t)\right) .
$$

Let $\overline{\mathbb{R}}_{+}=\mathbb{R}_{+} \cup\{0\}, \mathcal{Z}$ be a Banach space, $\mathcal{L}(\mathcal{Z})$ be the Banach space of all linear bounded operators on $\mathcal{Z}, \mathcal{C l}(\mathcal{Z})$ be the set of all linear closed operators, densely defined in $\mathcal{Z}$, acting into $\mathcal{Z}$. 
We shall write $A \in \mathcal{A}_{\alpha}\left(\theta_{0}, a_{0}\right)$ for some $\alpha>0, \theta_{0} \in(\pi / 2, \pi), a_{0} \geq 0$, if an operator $A \in \mathcal{C l}(\mathcal{Z})$ satisfies the following conditions:

(i) for every $\lambda \in S_{\theta_{0}, a_{0}}:=\left\{\mu \in \mathbb{C}:\left|\arg \left(\mu-a_{0}\right)\right|<\theta_{0}, \mu \neq a_{0}\right\}$ we have $\lambda^{\alpha} \in \rho(A):=\{\mu \in \mathbb{C}$ : $\left.(\mu I-A)^{-1} \in \mathcal{L}(\mathcal{Z})\right\}$

(ii) for any $a>a_{0}, \theta \in\left(\pi / 2, \theta_{0}\right)$ there exists $K=K(\theta, a)>0$, such that at all $\lambda \in S_{\theta, a}$

$$
\left\|\left(\lambda^{\alpha} I-A\right)^{-1}\right\|_{\mathcal{L}(\mathcal{Z})} \leq \frac{K(\theta, a)}{\left|\lambda^{\alpha-1}(\lambda-a)\right|} .
$$

Remark 1. It is known that at $\alpha \in(0,2)$ an operator $A \in \mathcal{C l}(\mathcal{Z})$ satisfies conditions (i) and (ii), if and only if there exists a resolving family of operators for the linear homogeneous equation $D_{t}^{\alpha} z(t)=A z(t)$ (see Theorem 2.14 [20], and more general Theorem I.2.1 [21]). Moreover, $A \in \mathcal{A}_{1}\left(\theta_{0}, a_{0}\right)$, if and only if it generates an analytic in a sector operator semigroup. In this case it is often called a sectorial operator.

Denote by $\partial S_{a, \theta}$ the boundary of $S_{a, \theta}:=\{\mu \in \mathbb{C}:|\arg (\mu-a)|<\theta, \mu \neq a\}$ at some $\theta \in\left(\pi / 2, \theta_{0}\right)$, $a>a_{0}$.

Lemma 1 ([22]). Let $\alpha>0, A \in \mathcal{A}_{\alpha}\left(\theta_{0}, a_{0}\right), \theta \in\left(\pi / 2, \theta_{0}\right), a>a_{0}$. Then the families of operators

$$
\left\{Z_{\beta}(t)=\frac{1}{2 \pi i} \int_{\partial S_{a, \theta}} \mu^{\alpha-1-\beta}\left(\mu^{\alpha} I-A\right)^{-1} e^{\mu t} d \mu \in \mathcal{L}(\mathcal{Z}): t \in \mathbb{R}_{+}\right\}, \beta \in \mathbb{R},
$$

admit analytic extensions to $\Sigma_{\theta_{0}}:=\left\{\tau \in \mathbb{C}:|\arg \tau|<\theta_{0}-\pi / 2, \tau \neq 0\right\}$.

Remark 2. It can be shown that for a bounded operator $A \in \mathcal{L}(\mathcal{Z})$ we have $Z_{\beta}(t)=t^{\beta} E_{\alpha, \beta+1}\left(t^{\alpha} A\right)$, where $E_{\alpha, \beta+1}$ is the Mittag-Leffler function.

Consider the Cauchy problem

$$
z^{(k)}(0)=z_{k}, \quad k=0,1, \ldots, m-1
$$

for the inhomogeneous equation

$$
D_{t}^{\alpha} z(t)=A z(t)+f(t), \quad t \in(0, T]
$$

where $A \in \mathcal{A}_{\alpha}\left(\theta_{0}, a_{0}\right), T>0, f:[0, T] \rightarrow \mathcal{Z}$. A solution of problem (4) and (5) is a function $z \in C\left((0, T] ; \mathcal{D}_{A}\right) \cap C^{m-1}([0, T] ; \mathcal{Z})$, such that

$$
g_{m-\alpha} *\left(z-\sum_{k=0}^{m-1} z^{(k)}(0) g_{k+1}\right) \in C^{m}((0, T] ; \mathcal{Z})
$$

and Equalities (4) and (5) for all $t \in(0, T]$ are satisfied.

Remark 3. It is known [20] that the resolving operators family for the homogeneous ( $f \equiv 0)$ Equation (5) is $\left\{Z_{0}(t): t \in \overline{\mathbb{R}}_{+}\right\}$, where $Z_{0}(0)=I$.

A mapping $f \in C([0, T] ; \mathcal{Z})$ is called Hölder function with a power $\gamma \in(0,1]$, if there exists a constant $C>0$, such that for all $t, s \in[0, T]$ we have $\|f(t)-f(s)\|_{\mathcal{Z}} \leq C|t-s|^{\gamma}$. Denote the linear space of such functions with a fixed $\gamma$ by $C^{\gamma}([0, T] ; \mathcal{Z})$. 
Theorem 1 ([23]). Let $\alpha>0, A \in \mathcal{A}_{\alpha}\left(\theta_{0}, a_{0}\right), f \in C^{\gamma}([0, T] ; \mathcal{Z})$ for some $\gamma \in(0,1]$. Then for any $z_{k} \in \mathcal{D}_{A}$, $k=0,1, \ldots, m-1$, there exists a unique solution of problem (4) and (5). It has the form

$$
z(t)=\sum_{k=0}^{m-1} Z_{k}(t) z_{k}+\int_{0}^{t} Z_{\alpha-1}(t-s) f(s) d s .
$$

Remark 4. Analogous result with $f \in C\left([0, T] ; \mathcal{D}_{A}\right)$ is obtained in [24]. The case of a bounded operator $A$ and $f \in C([0, T] ; \mathcal{Z})$ is studied in [25].

\section{Degenerate System Solvability}

We now obtain an existence and uniqueness theorem for the degenerate equation, which describes the dynamics of fractional order degenerate systems.

Let $\mathcal{X}, \mathcal{Y}$ be Banach spaces, $\mathcal{L}(\mathcal{X} ; \mathcal{Y})$ be the Banach space of all linear bounded operators from $\mathcal{X}$ into $\mathcal{Y}, \mathcal{C l}(\mathcal{X} ; \mathcal{Y})$ be the set of all linear closed densely defined in $\mathcal{X}$ operators, acting into the space $\mathcal{Y}$. Let $L, M \in \mathcal{C l}(\mathcal{X} ; \mathcal{Y})$, $\operatorname{ker} L \neq\{0\}$. The set of points $\mu \in \mathbb{C}$, such that the operator $\mu L-M$ : $\mathcal{D}_{L} \cap \mathcal{D}_{M} \rightarrow \mathcal{Y}$ is injective, and $(\mu L-M)^{-1} L \in \mathcal{L}(\mathcal{X}), L(\mu L-M)^{-1} \in \mathcal{L}(\mathcal{Y})$, is called $L$-resolvent set $\rho^{L}(M)$ of the operator $M$. Introduce denotations $R_{\mu}^{L}(M):=(\mu L-M)^{-1} L, L_{\mu}^{L}(M):=L(\mu L-M)^{-1}$.

Definition 1. Let $\alpha>0, L, M \in \mathcal{C} l(\mathcal{X} ; \mathcal{Y})$. We say that a pair of operators $(L, M)$ belongs to the class $\mathcal{H}_{\alpha}\left(\theta_{0}, a_{0}\right)$, if

(i) there exist $\theta_{0} \in(\pi / 2, \pi)$ and $a_{0} \geq 0$, such that for all $\lambda \in S_{\theta_{0}, a_{0}}$ inclusion $\lambda^{\alpha} \in \rho^{L}(M)$ is valid;

(ii) for any $\theta \in\left(\pi / 2, \theta_{0}\right), a>a_{0}$ there exists a constant $K=K(\theta, a)>0$, such that for all $\lambda \in S_{\theta, a}$

$$
\max \left\{\left\|R_{\lambda^{\alpha}}^{L}(M)\right\|_{\mathcal{L}(\mathcal{X})},\left\|L_{\lambda^{\alpha}}^{L}(M)\right\|_{\mathcal{L}(\mathcal{Y})}\right\} \leq \frac{K(\theta, a)}{\left|\lambda^{\alpha-1}(\lambda-a)\right|} .
$$

Remark 5. If there exists an inverse operator $L^{-1} \in \mathcal{L}(\mathcal{Y} ; \mathcal{X})$, then $(L, M) \in \mathcal{H}_{\alpha}\left(\theta_{0}, a_{0}\right)$, if and only if $L^{-1} M \in \mathcal{A}_{\alpha}\left(\theta_{0}, a_{0}\right)$ and $M L^{-1} \in \mathcal{A}_{\alpha}\left(\theta_{0}, a_{0}\right)$.

It is not difficult to show that the subspaces $\operatorname{ker} R_{\mu}^{L}(M)=\operatorname{ker} L, \operatorname{im} R_{\mu}^{L}(M), \operatorname{ker} L_{\mu}^{L}(M), \operatorname{im} L_{\mu}^{L}(M)$ do not depend on $\mu \in \rho^{L}(M)$. Introduce the denotations $\operatorname{ker} R_{\mu}^{L}(M):=\mathcal{X}^{0}, \operatorname{ker} L_{\mu}^{L}(M):=\mathcal{Y}^{0}$. By $\mathcal{X}^{1}$ $\left(\mathcal{Y}^{1}\right)$ we denote the closure of $\operatorname{im} R_{\mu}^{L}(M)\left(\operatorname{im} L_{\mu}^{L}(M)\right)$ in the norm of the space $\mathcal{X}(\mathcal{Y})$. By $L_{k}\left(M_{k}\right)$ the restriction of the operator $L(M)$ on $\mathcal{D}_{L_{k}}:=\mathcal{D}_{L} \cap \mathcal{X}^{k}\left(\mathcal{D}_{M_{k}}:=\mathcal{D}_{M} \cap \mathcal{X}^{k}\right)$ is denoted, $k=0,1$.

Theorem 2 ([22]). Let Banach spaces $\mathcal{X}$ and $\mathcal{Y}$ be reflexive, $(L, M) \in \mathcal{H}_{\alpha}\left(\theta_{0}, a_{0}\right)$. Then

(i) $\mathcal{X}=\mathcal{X}^{0} \oplus \mathcal{X}^{1}, \mathcal{Y}=\mathcal{Y}^{0} \oplus \mathcal{Y}^{1}$;

(ii) the projector $P(Q)$ on the subspace $\mathcal{X}^{1}\left(\mathcal{Y}^{1}\right)$ along $\mathcal{X}^{0}\left(\mathcal{Y}^{0}\right)$ has the form $P=s^{-} \lim _{n \rightarrow \infty} n R_{n}^{L}(M)(Q=$ $\left.s-\lim _{n \rightarrow \infty} n L_{n}^{L}(M)\right)$;

(iii) $L_{0}=0, M_{0} \in \mathcal{C l}\left(\mathcal{X}^{0} ; \mathcal{Y}^{0}\right), L_{1}, M_{1} \in \mathcal{C l}\left(\mathcal{X}^{1} ; \mathcal{Y}^{1}\right)$;

(iv) there exist inverse operators $L_{1}^{-1} \in \mathcal{C l}\left(\mathcal{Y}^{1} ; \mathcal{X}^{1}\right), M_{0}^{-1} \in \mathcal{L}\left(\mathcal{Y}^{0} ; \mathcal{X}^{0}\right)$;

(v) $\forall x \in \mathcal{D}_{L} P x \in \mathcal{D}_{L}$ and $L P x=Q L x$;

(vi) $\forall x \in \mathcal{D}_{M} P x \in \mathcal{D}_{M}$ and $M P x=Q M x$;

(vii) let $S:=L_{1}^{-1} M_{1}: \mathcal{D}_{S} \rightarrow \mathcal{X}^{1}$, then $\mathcal{D}_{S}:=\left\{x \in \mathcal{D}_{M_{1}}: M_{1} x \in \operatorname{im} L_{1}\right\}$ is dense in $\mathcal{X}$;

(viii) let $V:=M_{1} L_{1}^{-1}: \mathcal{D}_{V} \rightarrow \mathcal{Y}^{1}$, then $\mathcal{D}_{V}:=\left\{y \in \operatorname{imL} L_{1}: L_{1}^{-1} y \in \mathcal{D}_{M_{1}}\right\}$ is dense in $\mathcal{Y}$;

(ix) if $L_{1} \in \mathcal{L}\left(\mathcal{X}^{1} ; \mathcal{Y}^{1}\right)$, or $M_{1} \in \mathcal{L}\left(\mathcal{X}^{1} ; \mathcal{Y}^{1}\right)$, then $S \in \mathcal{A}_{\alpha}\left(\theta_{0}, a_{0}\right)$;

(x) if $L_{1}^{-1} \in \mathcal{L}\left(\mathcal{Y}^{1} ; \mathcal{X}^{1}\right)$, or $M_{1}^{-1} \in \mathcal{L}\left(\mathcal{Y}^{1} ; \mathcal{X}^{1}\right)$, then $V \in \mathcal{A}_{\alpha}\left(\theta_{0}, a_{0}\right)$; 
(xi) the families of operators

$$
\begin{aligned}
& \left\{X_{\beta}(t)=\frac{1}{2 \pi i} \int_{\partial S_{a, \theta}} \mu^{\alpha-1-\beta} R_{\mu^{\alpha}}^{L}(M) e^{\mu t} d \mu \in \mathcal{L}(\mathcal{X}): t \in \mathbb{R}_{+}\right\}, \beta \in \mathbb{R}, \\
& \left\{Y_{\beta}(t)=\frac{1}{2 \pi i} \int_{\partial S_{a, \theta}} \mu^{\alpha-1-\beta} L_{\mu^{\alpha}}^{L}(M) e^{\mu t} d \mu \in \mathcal{L}(\mathcal{Y}): t \in \mathbb{R}_{+}\right\}, \beta \in \mathbb{R},
\end{aligned}
$$

admit analytic extensions to $\Sigma_{\theta_{0}}:=\left\{t \in \mathbb{C}:|\arg t|<\theta_{0}-\pi / 2, t \neq 0\right\}$. For any $\theta \in\left(\pi / 2, \theta_{0}\right)$, $a>a_{0}$ there exists such $C_{\beta}=C_{\beta}(\theta, a)$, that for each $t \in \Sigma_{\theta}$

$$
\begin{gathered}
\max \left\{\left\|X_{\beta}(t)\right\|_{\mathcal{L}(\mathcal{X})},\left\|Y_{\beta}(t)\right\|_{\mathcal{L}(\mathcal{Y})}\right\} \leq C_{\beta}(\theta, a) e^{a \operatorname{Re} t}\left(|t|^{-1}+a\right)^{-\beta}, \quad \beta \leq 0, \\
\max \left\{\left\|X_{\beta}(t)\right\|_{\mathcal{L}(\mathcal{X})},\left\|Y_{\beta}(t)\right\|_{\mathcal{L}(\mathcal{Y})}\right\} \leq C_{\beta}(\theta, a) e^{a \operatorname{Re} t}|t|^{\beta}, \quad \beta>0 .
\end{gathered}
$$

Consider the degenerate $(\operatorname{ker} L \neq\{0\})$ inhomogeneous equation

$$
D_{t}^{\alpha} L x(t)=M x(t)+f(t), \quad t \in(0, T]
$$

with a given $f:[0, T] \rightarrow \mathcal{Y}$. Its solution is a function $x \in C\left((0, T] ; \mathcal{D}_{M}\right)$, such that $L x \in C^{m-1}([0, T] ; \mathcal{Y})$, $g_{m-\alpha} *\left(L x-\sum_{k=0}^{m-1}(L x)^{(k)}(0) g_{k+1}\right) \in C^{m}((0, T] ; \mathcal{Y})$, and for all $t \in(0, T]$ equality (8) is fulfilled. A solution of the Cauchy problem

$$
x^{(k)}(0)=x_{k}, \quad k=0,1, \ldots, m-1
$$

for Equation (8) is a solution of the equation, such that $x \in C^{m-1}([0, T] ; \mathcal{X})$ and conditions (9) are satisfied.

Theorem 3. Let $\alpha>0$, Banach spaces $\mathcal{X}, \mathcal{Y}$ be reflexive, $(L, M) \in \mathcal{H}_{\alpha}\left(\theta_{0}, a_{0}\right), L_{1} \in \mathcal{L}\left(\mathcal{X}^{1} ; \mathcal{Y}^{1}\right)$ or $M_{1} \in \mathcal{L}\left(\mathcal{X}^{1} ; \mathcal{Y}^{1}\right), f:[0, T] \rightarrow \mathcal{Y}^{0} \dot{+i m} L_{1}$, at some $\gamma \in(0,1] L_{1}^{-1} Q f \in C^{\gamma}([0, T] ; \mathcal{X}),(I-Q) f \in$ $C^{m-1}([0, T] ; \mathcal{Y}), x_{k} \in \mathcal{D}_{M}, P x_{k} \in \mathcal{D}_{S}, k=0,1, \ldots, m-1$, equalities

$$
\left.D_{t}^{k}\right|_{t=0} M_{0}^{-1}(I-Q) f(t)=-(I-P) x_{k}, \quad k=0,1, \ldots, m-1 \text {, }
$$

are valid. Then there exists a unique solution of problem (8) and (9), moreover, it has the form

$$
x(t)=\sum_{k=0}^{m-1} X_{k}(t) x_{k}+\int_{0}^{t} X_{\alpha-1}(t-s) L_{1}^{-1} Q f(s) d s-M_{0}^{-1}(I-Q) f(t) .
$$

Proof. Put $x^{0}(t):=(I-P) x(t), x^{1}(t):=P x(t)$. By virtue of Theorem 2 Equation (8) can be reduced to the system of the two equations

$$
\begin{gathered}
0=x^{0}(t)+M_{0}^{-1}(I-Q) f(t), \\
D_{t}^{\alpha} x^{1}(t)=S x^{1}(t)+g(t), \quad S:=L_{1}^{-1} M_{1}, \quad g(t):=L_{1}^{-1} Q f(t) .
\end{gathered}
$$

Therefore, $x^{0}(t)=-M_{0}^{-1}(I-Q) f(t)$, and for the satisfying of Cauchy conditions (9) it is necessarry the fulfillment of (10). Due to Theorem $2 S \in \mathcal{A}_{\alpha}\left(\theta_{0}, a_{0}\right)$, hence Theorem 1 implies 
the existence of a unique solution of the Cauchy problem $x^{1(k)}(0)=P x_{k}, k=0,1, \ldots, m-1$, to Equation (12). Besides,

$$
\begin{gathered}
x^{1}(t)=\frac{1}{2 \pi i} \sum_{k=0}^{m-1} \int_{\partial S_{a, \theta}} \mu^{\alpha-k-1}\left(\mu^{\alpha} I-S\right)^{-1} e^{\mu t} d \mu P x_{k}+\frac{1}{2 \pi i} \int_{0}^{t} \int_{\partial S_{a, \theta}}\left(\mu^{\alpha} I-S\right)^{-1} e^{\mu(t-s)} d \mu g(s) d s= \\
=\frac{1}{2 \pi i} \sum_{k=0}^{m-1} \int_{\partial S_{a, \theta}} \mu^{\alpha-k-1}\left(\mu^{\alpha} L-M\right)^{-1} L e^{\mu t} d \mu x_{k}+\frac{1}{2 \pi i} \int_{0}^{t} \int_{\partial S_{a, \theta}}\left(\mu^{\alpha} L-M\right)^{-1} L e^{\mu(t-s)} d \mu g(s) d s,
\end{gathered}
$$

since $L(I-P)=0$, the operator $\left(\lambda L_{0}-M_{0}\right)^{-1}=-M_{0}^{-1}$ exists for every $\lambda \in \mathbb{C}$.

Theorem 4. Let $\alpha>0$, Banach spaces $\mathcal{X}, \mathcal{Y}$ be reflexive, $(L, M) \in \mathcal{H}_{\alpha}\left(\theta_{0}, a_{0}\right), L_{1}^{-1} \in \mathcal{L}\left(\mathcal{Y}^{1} ; \mathcal{X}^{1}\right)$ or $M_{1}^{-1} \in \mathcal{L}\left(\mathcal{Y}^{1} ; \mathcal{X}^{1}\right), f \in C([0, T] ; \mathcal{Y}), Q f \in C^{\gamma}([0, T] ; \mathcal{Y})$ at some $\gamma \in(0,1],(I-Q) f \in C^{m-1}([0, T] ; \mathcal{Y})$, $x_{k} \in \mathcal{D}_{M}, P x_{k} \in \mathcal{D}_{L}, k=0,1, \ldots, m-1$, equalities (10) are valid. Then there exists a unique solution of problem (8) and (9), and it has form (11).

Proof. In this case, instead of Equation (12) we obtain the equation

$$
D_{t}^{\alpha} y(t)=V y(t)+h(t), \quad V:=M_{1} L_{1}^{-1}, \quad h(t):=Q f(t),
$$

where $y(t):=L_{1} x^{1}(t)=L_{1} P x(t)$. Theorem 2 implies, that $V \in \mathcal{A}_{\alpha}\left(\theta_{0}, a_{0}\right)$, and due to Theorem 1 there exists a unique solution of the Cauchy problem $y^{(k)}(0)=L_{1} P x_{k} \in \mathcal{D}_{V}, k=0,1, \ldots, m-1$, for Equation (13). The solution has the form

$$
\begin{gathered}
y(t)=\frac{1}{2 \pi i} \sum_{k=0}^{m-1} \int_{\partial S_{a, \theta}} \mu^{\alpha-k-1}\left(\mu^{\alpha} I-V\right)^{-1} e^{\mu t} d \mu L_{1} P x_{k}+\frac{1}{2 \pi i} \int_{0}^{t} \int_{\partial S_{a, \theta}}\left(\mu^{\alpha} I-V\right)^{-1} e^{\mu(t-s)} d \mu h(s) d s= \\
=\frac{1}{2 \pi i} \sum_{k=0}^{m-1} Y_{k}(t) L_{1} P x_{k}+\int_{0}^{t} Y_{\alpha-1}(t-s) Q f(s) d s,
\end{gathered}
$$

therefore, $x^{1}(t)=L_{1}^{-1} y(t)$ has form (11). The function $x^{0}(t)$ is the same as in the previous proof.

So, the Cauchy problem for degenerate Equation (8) is overdetermined due to the necessity of conditions (10). Consider the so-called Showalter-Sidorov problem

$$
(L x)^{(k)}(0)=y_{k}, \quad k=0,1, \ldots, m-1,
$$

which is natural for weakly degenerate evolution equations, when the degeneration subspace $\mathcal{X}^{0}$ coincides with $\operatorname{ker} L$. A solution of this problem to Equation (8) is a solution of the equation, such that conditions (15) are satisfied.

Reasoning as before, we can prove the next assertions.

Theorem 5. Let $\alpha>0$, Banach spaces $\mathcal{X}, \mathcal{Y}$ be reflexive, $(L, M) \in \mathcal{H}_{\alpha}\left(\theta_{0}, a_{0}\right), L_{1} \in \mathcal{L}\left(\mathcal{X}^{1} ; \mathcal{Y}^{1}\right)$ or $M_{1} \in \mathcal{L}\left(\mathcal{X}^{1} ; \mathcal{Y}^{1}\right), f \in C([0, T] ; \mathcal{Y}), Q f(t) \in \operatorname{imL}$ for all $t \in[0, T], L_{1}^{-1} Q f \in C^{\gamma}([0, T] ; \mathcal{X})$ at some $\gamma \in(0,1], y_{k} \in L\left[\mathcal{D}_{L} \cap \mathcal{D}_{M}\right], L_{1}^{-1} y_{k} \in \mathcal{D}_{S}, k=0,1, \ldots, m-1$. Then there exists a unique solution of problem (8) and (15), and it has form

$$
x(t)=\sum_{k=0}^{m-1} X_{k}(t) L_{1}^{-1} y_{k}+\int_{0}^{t} X_{\alpha-1}(t-s) L_{1}^{-1} Q f(s) d s-M_{0}^{-1}(I-Q) f(t) .
$$


Theorem 6. Let $\alpha>0$, Banach spaces $\mathcal{X}, \mathcal{Y}$ be reflexive, $(L, M) \in \mathcal{H}_{\alpha}\left(\theta_{0}, a_{0}\right), L_{1}^{-1} \in \mathcal{L}\left(\mathcal{Y}^{1} ; \mathcal{X}^{1}\right)$ or $M_{1}^{-1} \in \mathcal{L}\left(\mathcal{Y}^{1} ; \mathcal{X}^{1}\right), f \in C([0, T] ; \mathcal{Y}), Q f \in C^{\gamma}([0, T] ; \mathcal{Y})$ for some $\gamma \in(0,1], y_{k} \in L\left[\mathcal{D}_{L} \cap \mathcal{D}_{M}\right], k=$ $0,1, \ldots, m-1$. Then there exists a unique solution of problem (8) and (15), and it has form (16).

Here, in contrast to the proofs of Theorems 3 and 4 we have no initial conditions for $(I-Q) x(t)$ and there is not condition $(I-Q) f \in C^{m-1}([0, T] ; \mathcal{Y})$ nor condition (10) of the matching of initial data with the right-hand side of Equation (8).

Remark 6. Note that, due to Theorem $2 L=L_{1} P+0(I-P)=L_{1} P$, therefore, $\operatorname{im} L=\operatorname{im} L_{1} \subset \mathcal{Y}^{1}$. Thus, $y_{k} \in L\left[\mathcal{D}_{L} \cap \mathcal{D}_{M}\right]=L_{1}\left[\mathcal{D}_{L_{1}} \cap \mathcal{D}_{M_{1}}\right]$, if and only if $y_{k} \in \mathcal{D}_{V}$. So, under the conditions of Theorem 6 the set $L\left[\mathcal{D}_{L} \cap \mathcal{D}_{M}\right]=\mathcal{D}_{V}$ is dense in $\mathcal{Y}^{1}$.

Remark 7. Study of the degenerate system controllability will be carried out in the next sections on the basis of Theorem 6 , since its conditions on $f$ and $y_{k}$ are less restrictive than those in Theorem 5.

Remark 8. It can be shown that in the case of reflexive Banach spaces $\mathcal{X}$ and $\mathcal{Y}$ for $(L, M) \in \mathcal{H}_{\alpha}\left(\theta_{0}, a_{0}\right)$ conditions (15) are equivalent to the conditions $(P x)^{(k)}(0)=L_{1}^{-1} y_{k}, k=0,1, \ldots, m-1$. Recall that $\operatorname{im} L \subset \mathcal{X}^{1}$.

\section{Approximate Controllability of Subsystems}

Here, we reduce the degenerate control system to two subsystems on mutually complement subspaces.

Let $\mathcal{X}, \mathcal{Y}$ be reflexive Banach spaces, $\mathcal{U}$ be a Banach space, $L, M \in \mathcal{C l}(\mathcal{X} ; \mathcal{Y}),(L, M) \in \mathcal{H}_{\alpha}\left(\theta_{0}, a_{0}\right)$. Denote by $C_{Q}^{\gamma}([0, T] ; \mathcal{L}(\mathcal{U} ; \mathcal{Y}))$ for some $\gamma \in(0,1]$ the linear space of all operator-valued functions $B \in C([0, T] ; \mathcal{L}(\mathcal{U} ; \mathcal{Y}))$, such that $Q B \in C^{\gamma}([0, T] ; \mathcal{L}(\mathcal{U} ; \mathcal{Y}))$. Analogously, $C_{Q}^{\gamma}([0, T] ; \mathcal{Y})$ is the set of all vector-valued functions $g \in C([0, T] ; \mathcal{Y})$, such that $Q g \in C^{\gamma}([0, T] ; \mathcal{Y})$.

Further, we shall assume that $B \in C_{Q}^{\gamma}([0, T] ; \mathcal{L}(\mathcal{U} ; \mathcal{Y})), g \in C_{Q}^{\gamma}([0, T] ; \mathcal{Y})$ for some $\gamma \in(0,1]$. Control functions $u(\cdot)$ for the system, which is described by the Showalter-Sidorov problem

$$
\begin{aligned}
& (L x)^{(k)}(0)=y_{k}, \quad k=0,1, \ldots, m-1, \\
& D_{t}^{\alpha} L x(t)=M x(t)+B(t) u(t)+g(t),
\end{aligned}
$$

will be choosen from the space $C^{\gamma}([0, T] ; \mathcal{U})$, hence $B u \in C_{Q}^{\gamma}([0, T] ; \mathcal{Y})$. By means of Theorem 2 problem (17) and (18) can be reduced to the initial value problem

$$
y^{(k)}(0)=y_{k}, \quad k=0,1, \ldots, m-1
$$

for the system of equations

$$
\begin{gathered}
D_{t}^{\alpha} y(t)=V y(t)+Q B(t) u(t)+Q g(t), \\
x^{0}(t)=-M_{0}^{-1}(I-Q)(B(t) u(t)+g(t))
\end{gathered}
$$

on the subspaces $\mathcal{Y}^{1}$ and $\mathcal{X}^{0}$, respectively. Here $V=M_{1} L_{1}^{-1} \in \mathcal{C l}\left(\mathcal{Y}^{1}\right), y(t)=L_{1} P x(t), x^{0}(t)=$ $(I-P) x(t)$. Note that due to Theorem 1 the solution of problem (19) and (20) has the form

$$
y(t)=\sum_{k=0}^{m-1} Y_{k}(t) y_{k}+\int_{0}^{t} Y_{\alpha-1}(t-s) Q(B(s) u(s)+g(s)) d s
$$

Denoted by $x(T ; \bar{y} ; u)$, the value at the time moment $T$ of the solution to problem (17) and (18) with the initial data $\bar{y}=\left(y_{0}, y_{1}, \ldots, y_{m-1}\right)$ in (17) and with a control function $u$. Denoted by $y(T ; \bar{y} ; u)$, 
the value at the time $T$ of the solution for the subsystem, described by (19), (20). And by $x^{0}(T ; u)$ denotes the value at $t=T$ of function (21).

System (18) is called approximately controllable in time $T>0$, if, for every $\varepsilon>0, \hat{x} \in \mathcal{X}$, $\bar{y}=\left(y_{0}, y_{1}, \ldots, y_{m-1}\right) \in\left(L\left[\mathcal{D}_{L} \cap \mathcal{D}_{M}\right]\right)^{m}$ in (17) there exists a control function $u \in C^{\gamma}([0, T] ; \mathcal{U})$, such that $\|x(T ; \bar{y} ; u)-\hat{x}\|_{\mathcal{X}} \leq \varepsilon$.

System (20) is called approximately controllable in time $T>0$, if for all $\varepsilon>0, \hat{y} \in \mathcal{Y}^{1}$, $\bar{y}=\left(y_{0}, y_{1}, \ldots, y_{m-1}\right) \in\left(\mathcal{D}_{V}\right)^{m}$ in (19) there exists a control function $u \in C^{\gamma}([0, T] ; \mathcal{U})$, such that $\|y(T ; \bar{y} ; u)-\hat{y}\|_{\mathcal{Y}^{1}} \leq \varepsilon$.

System (21) is called approximately controllable in time $T>0$, if for every $\varepsilon>0, \hat{x}^{0} \in \mathcal{X}^{0}$ there exists $u \in C([0, T] ; \mathcal{U})$, such that $\left\|x^{0}(T ; u)-\hat{x}^{0}\right\|_{\mathcal{X}^{0}} \leq \varepsilon$.

Remark 9. We take u not from $C^{\gamma}([0, T] ; \mathcal{U})$ in the last definition, since due to the definition of problem (8) and (15) solution, the continuity of $u$ is sufficient for the existence of the subsystem (21) solution, since $x^{0}(t) \in \operatorname{ker} L$ for all $t$.

The following result shows that, while controlling two systems (20) and (21) by the same function $u(\cdot)$, we can, nevertheless, simultaneously lead the trajectories of the both systems into the $\varepsilon$-neighborhood of respective given points $\hat{y} \in \mathcal{Y}^{1}, \hat{x}^{0} \in \mathcal{X}^{0}$.

Theorem 7. Let Banach spaces $\mathcal{X}, \mathcal{Y}$ be reflexive, $(L, M) \in \mathcal{H}_{\alpha}\left(\theta_{0}, a_{0}\right), L_{1}^{-1} \in \mathcal{L}\left(\mathcal{Y}^{1} ; \mathcal{X}^{1}\right), B \in$ $C_{Q}^{\gamma}([0, T] ; \mathcal{L}(\mathcal{U} ; \mathcal{Y})), g \in C_{Q}^{\gamma}([0, T] ; \mathcal{Y})$ for some $\gamma \in(0,1]$. Then system $(18)$ is approximately controllable in time $T$, if and only if its subsystems (20) and (21) are approximately controllable in time $T$.

Proof. The direct assertion of Theorem 7 is obvious, since system (18) splits into two mutually complementary subsystems (20) and (21). Consider the inverse assertion of Theorem 7. Let for all $\hat{x}^{0} \in \mathcal{X}^{0}, \varepsilon>0$ there exists a function $u^{0} \in C([0, T] ; \mathcal{U})$, such that

$$
\left\|-M_{0}^{-1}(I-Q)\left(B(T) u^{0}(T)+g(T)\right)-\hat{x}^{0}\right\|_{\mathcal{X}} \leq \varepsilon / 3,
$$

and

$$
\forall \bar{y} \in\left(\mathcal{D}_{T}\right)^{m} \forall \hat{y} \in \mathcal{Y}^{1} \forall \varepsilon>0 \exists u^{1} \in C^{\gamma}([0, T] ; \mathcal{U})\left\|y\left(T ; \bar{y} ; u^{1}\right)-\hat{y}\right\|_{\mathcal{Y}} \leq \varepsilon /\left(3\left\|L_{1}^{-1}\right\|_{\mathcal{L}\left(\mathcal{Y}^{1} ; \mathcal{X}^{1}\right)}\right) .
$$

Then choose the new control function $u$, such that $u(t)=u^{1}(t)$ at $t \in[0, \delta]$ for some $\delta \in(T / 2, T)$, and $u(t)=u^{1}(\delta)+\gamma(t-\delta)+b(t-\delta)^{2}$ at $t \in(\delta, T]$ with

$$
\gamma=\frac{d u^{1}}{d t}(\delta), \quad b=\frac{u^{0}(T)-u^{1}(\delta)-\gamma(T-\delta)}{(T-\delta)^{2}}
$$

Then

$$
u(t)=u^{1}(\delta)+\gamma(t-\delta)+\frac{(t-\delta)^{2}}{(T-\delta)^{2}}\left(u^{0}(T)-u^{1}(\delta)-\gamma(T-\delta)\right) \in C^{\gamma}([0, T] ; \mathcal{U}), \quad u(T)=u^{0}(T)
$$

Note that for any $\delta \in(T / 2, T)$

$$
\|u(t)\|_{\mathcal{U}} \leq C:=2 \max _{t \in[0, T]}\left\|\mathcal{u}^{1}(t)\right\|_{\mathcal{U}}+\|\gamma\|_{\mathcal{U}} T+\left\|u^{0}(T)\right\|_{\mathcal{U}}, \quad t \in[0, T]
$$

where $C$ is independent of $\delta$. 
For arbitrary $\hat{x} \in \mathcal{X}$ take the control function, constructed as it was explained before with $\hat{x}^{0}=(I-P) \hat{x}$ and $\hat{y}=L \hat{x}$, then for sufficiently small $T-\delta>0$,

$$
\begin{gathered}
\|x(T ; \bar{y} ; u)-\hat{x}\|_{\mathcal{X}} \leq\left\|x^{0}\left(T ; u^{0}\right)-(I-P) \hat{x}\right\|_{\mathcal{X}}+\left\|L_{1}^{-1} y\left(T ; \bar{y} ; u^{1}\right)-L_{1}^{-1} L \hat{x}\right\|_{\mathcal{X}}+ \\
+\left\|L_{1}^{-1} y(T ; \bar{y} ; u)-L_{1}^{-1} y\left(T ; \bar{y} ; u^{1}\right)\right\| \mathcal{X} \leq 2 \varepsilon / 3+2 C\left\|L_{1}^{-1}\right\|_{\mathcal{L}\left(\mathcal{Y}^{1} ; \mathcal{X}^{1}\right)} \int_{\delta}^{T}\left\|Y_{\alpha-1}(T-s) Q B(s)\right\|_{\mathcal{L}(\mathcal{U} ; \mathcal{Y})} d s \leq \varepsilon
\end{gathered}
$$

Here, we take into account estimate (7) for $\alpha>1$. At $\alpha \in(0,1]$ due to (6) we also have

$$
\int_{\delta}^{T}\left\|Y_{\alpha-1}(T-s) Q B(s)\right\|_{\mathcal{L}(\mathcal{U} ; \mathcal{Y})} d s \leq C_{1}(1+a T)^{1-\alpha}(T-\delta)^{\alpha} \rightarrow 0 \text { as } \delta \rightarrow T-
$$

Analogously, the notion of the approximate controllability in free time can be defined. For example, system (18) is called approximately controllable in free time, if for every $\varepsilon>0, \hat{x} \in \mathcal{X}, \bar{y}=$ $\left(y_{0}, y_{1}, \ldots, y_{m-1}\right) \in\left(L\left[\mathcal{D}_{L} \cap \mathcal{D}_{M}\right]\right)^{m}$ in (17) there exists $T>0$ and a control function $u \in C^{\gamma}([0, T] ; \mathcal{U})$, $\gamma \in(0,1]$, such that $\|x(T ; \bar{y} ; u)-\hat{x}\|_{\mathcal{X}} \leq \varepsilon$.

Theorem 8. Let Banach spaces $\mathcal{X}, \mathcal{Y}$ be reflexive, $(L, M) \in \mathcal{H}_{\alpha}\left(\theta_{0}, a_{0}\right), L_{1}^{-1} \in \mathcal{L}\left(\mathcal{Y}^{1} ; \mathcal{X}^{1}\right)$, for every $T>0 B \in C_{Q}^{\gamma(T)}([0, T] ; \mathcal{L}(\mathcal{U} ; \mathcal{Y})), g \in C_{Q}^{\gamma(T)}([0, T] ; \mathcal{Y}), \gamma(T) \in(0,1]$. Then system $(18)$ is approximately controllable in free time, if and only if its subsystems (20) and (21) are approximately controllable in free time.

Proof. This statement can be proved as Theorem 7. Let us prove the inverse assertion. Let $\varepsilon>0, \hat{x} \in \mathcal{X}$, $\bar{y}=\left(y_{0}, y_{1}, \ldots, y_{m-1}\right) \in\left(L\left[\mathcal{D}_{L} \cap \mathcal{D}_{M}\right]\right)^{m}$ and there exist $T_{1}>0, u^{1} \in C^{\gamma\left(T_{1}\right)}\left(\left[0, T_{1}\right] ; \mathcal{U}\right)$, such that $\left\|y\left(T_{1} ; \bar{y} ; u^{1}\right)-L \hat{x}\right\|_{\mathcal{Y}^{1}} \leq \varepsilon / 3$, and $T_{0}>0, u^{0} \in C\left(\left[0, T_{0}\right] ; \mathcal{U}\right)$, such that $\left\|x^{0}\left(T_{0} ; u^{0}\right)-(I-P) \hat{x}\right\|_{\mathcal{X}^{0}} \leq \varepsilon / 3$. Take the control function $u$ as in the proof of Theorem 7 with $T=T_{1}$, then $\left\|x\left(T_{1} ; \bar{y} ; u\right)-\hat{x}\right\|_{\mathcal{Y}^{1}} \leq \varepsilon$.

\section{Criterion of Approximate Controllability}

Now let us obtain a criterion of the fractional order degenerate control system approximate controllability in terms of the operators from the respective equation.

Let $\mathcal{Z}$ be a Banach space, $\mathcal{A}$ be some set of indices, $\alpha \in \mathcal{A}, \mathcal{D}_{\alpha} \subset \mathcal{Z}$. By $\operatorname{span}\left\{\mathcal{D}_{\alpha}: \alpha \in \mathcal{A}\right\}$ we denote the linear span of the sets $\mathcal{D}_{\alpha}$ union, $\alpha \in \mathcal{A}$, and by $\overline{\operatorname{span}}\left\{\mathcal{D}_{\alpha}: \alpha \in \mathcal{A}\right\}$ its closure in the space $\mathcal{Z}$ is denoted. We denote by $\overline{\operatorname{im}} A$ the closure of the image $\operatorname{im} A$ of an operator $A: \mathcal{D}_{A} \rightarrow \mathcal{Z}$.

Lemma 2. Let Banach spaces $\mathcal{X}, \mathcal{Y}$ be reflexive, $(L, M) \in \mathcal{H}_{\alpha}\left(\theta_{0}, a_{0}\right), L_{1}^{-1} \in \mathcal{L}\left(\mathcal{Y}^{1} ; \mathcal{X}^{1}\right), Q B \in$ $C^{\gamma}([0, T] ; \mathcal{L}(\mathcal{U} ; \mathcal{Y})), Q g \in C^{\gamma}([0, T] ; \mathcal{Y})$ for some $\gamma \in(0,1]$. Then system $(20)$ is approximately controllable in time $T$, if and only if

$$
\overline{\operatorname{span}}\left\{\operatorname{im} Y_{\alpha-1}(T-s) Q B(s): 0<s<T\right\}=\mathcal{Y}^{1}
$$

Proof. Form (22) of the Cauchy problem solution implies that it is sufficient to consider only the approximate controllability of system $(20)$ from zero $(\bar{y}=0)$. Suppose that the system is not approximately controllable from zero. Then the set of vectors of the form

$$
\int_{0}^{T} Y_{\alpha-1}(T-s) Q B(s) u(s) d s, \quad u \in C^{\gamma}([0, T] ; \mathcal{U})
$$


is not dense in the space $\mathcal{Y}^{1}$. By the Hahn-Banach Theorem, in this case there exists $f \in \mathcal{Y}^{1^{*}} \backslash\{0\}$, such that

$$
f\left(\int_{0}^{T} Y_{\alpha-1}(T-s) Q B(s) u(s) d s\right)=\int_{0}^{T} f\left(Y_{\alpha-1}(T-s) Q B(s) u(s)\right) d s=0
$$

for all $u \in C^{\gamma}([0, T] ; \mathcal{U})$.

For every $v$ from the Lebesgue-Bochner space $L_{p}(0, T ; \mathcal{U}), \max \{1,1 / \alpha\}<p<\infty$, there exists a sequence $\left\{u_{n}\right\} \subset C^{\gamma}([0, T] ; \mathcal{U})$, such that $\lim _{n \rightarrow \infty} u_{n}=v$ in $L_{p}(0, T ; \mathcal{U})$. Therefore, using reasoning as in the end of Theorem 7 proof, i.e., applying inequalities (6) and (7), obtain

$$
\left|\int_{0}^{T} f\left(Y_{\alpha-1}(T-s) Q B(s)\left(u_{n}(s)-v(s)\right)\right) d s\right| \leq C\|f\|_{\mathcal{Y}^{1^{*}}} \int_{0}^{T} s^{(\alpha-1) p^{\prime}} d s \int_{0}^{T}\left\|u_{n}(s)-v(s)\right\|_{\mathcal{U}}^{p} d s \rightarrow 0
$$

as $n \rightarrow \infty$. Here we take into account, that inequality $p>1 / \alpha$ implies that $(\alpha-1) p^{\prime}+1>0$, where $p^{\prime}=p /(p-1)$. Consequently, equality (24) is valid for all $u \in L_{p}(0, T ; \mathcal{U})$.

Take $t_{0} \in(0, T)$ and small $\delta>0, u_{\delta}(t)=w \in \mathcal{U}$ at $t \in\left[t_{0}-\delta, t_{0}+\delta\right], u_{\delta}(t)=0$ for $t \in$ $[0, T] \backslash\left[t_{0}-\delta, t_{0}+\delta\right]$. Then $u_{\delta} \in L_{p}(0, T ; \mathcal{U})$, and by the continuity of the integrand

$$
0=\frac{1}{2 \delta} \int_{t_{0}-\delta}^{t_{0}+\delta} f\left(Y_{\alpha-1}(T-s) Q B(s) w\right) d s=f\left(Y_{\alpha-1}(T-\xi) Q B(\xi) w\right)
$$

for some $\xi \in\left(t_{0}-\delta, t_{0}+\delta\right)$. We pass to the limit as $\delta \rightarrow 0+$ and obtain the equality $f\left(Y_{\alpha-1}\left(T-t_{0}\right) Q B\left(t_{0}\right) w\right)=0$ for all $t_{0} \in(0, T), w \in \mathcal{U}$. Hence condition (23) is not satisfied.

The inverse statement is obvious due to the integral form (22) of the solution of Equation (20) with zero initial data.

This assertion can be formulated in terms of Section 2 in the next form.

Theorem 9. Let $A \in \mathcal{A}_{\alpha}\left(\theta_{0}, a_{0}\right), B \in C^{\gamma}([0, T] ; \mathcal{L}(\mathcal{U} ; \mathcal{Z})), g \in C^{\gamma}([0, T] ; \mathcal{Z})$ for some $\gamma \in(0,1]$. Then the system $D_{t}^{\alpha} z(t)=A z(t)+B(t) u(t)+g(t)$ is approximately controllable in time $T$, if and only if

$$
\overline{\operatorname{span}}\left\{\operatorname{imZ}_{\alpha-1}(T-s) B(s): 0<s<T\right\}=\mathcal{Z} .
$$

Remark 10. If $Q B(t)$ does not depend on $t$, then the approximate controllability of system (20) in time $T$ implies its approximate controllability in any time $T_{1}>T$, since

$$
\overline{\operatorname{span}}\left\{\operatorname{im} Y_{\alpha-1}(s) Q B: 0<s<T\right\} \subset \overline{\operatorname{span}}\left\{\operatorname{im} Y_{\alpha-1}(s) Q B: 0<s<T_{1}\right\} .
$$

The criterion of system (21) approximate controllability is obvious.

Lemma 3. Let Banach spaces $\mathcal{X}, \mathcal{Y}$ be reflexive, $(L, M) \in \mathcal{H}_{\alpha}\left(\theta_{0}, a_{0}\right)$, moreover, $(I-Q) B \in$ $C([0, T] ; \mathcal{L}(\mathcal{U} ; \mathcal{Y}))$ and $(I-Q) g \in C([0, T] ; \mathcal{Y})$. Then system $(21)$ is approximately controllable in time $T$, if and only if $\overline{\operatorname{im}} M_{0}^{-1}(I-Q) B(T)=\mathcal{X}^{0}$.

Remark 11. If $(I-Q) B(t)$ does not depend on $t$, then the approximate controllability of system (21) in time $T$ implies its approximate controllability at any time $T_{1}>0$.

Theorem 10. Let Banach spaces $\mathcal{X}, \mathcal{Y}$ be reflexive, $(L, M) \in \mathcal{H}_{\alpha}\left(\theta_{0}, a_{0}\right), L_{1}^{-1} \in \mathcal{L}\left(\mathcal{Y}^{1} ; \mathcal{X}^{1}\right), B \in$ $C_{Q}^{\gamma}([0, T] ; \mathcal{L}(\mathcal{U} ; \mathcal{Y})), g \in C_{Q}^{\gamma}([0, T] ; \mathcal{Y})$ for some $\gamma \in(0,1]$. Then system $(18)$ is approximately controllable in time $T$, if and only if $\overline{\operatorname{im}} M_{0}^{-1}(I-Q) B(T)=\mathcal{X}^{0}, \overline{\operatorname{span}}\left\{\operatorname{im} Y_{\alpha-1}(T-s) Q B(s): 0<s<T\right\}=\mathcal{Y}^{1}$. 
Proof. The required result follows from Theorem 7, Lemmas 2 and 3.

Remark 12. By Remarks 10, 11 and Theorem 10, if $B(t)$ does not depend on $t$, then the approximate controllability of system (18) in time $T$ implies its approximate controllability in any greater time $T_{1}>T$.

Similar result for the controllability in free time can be obtained analogously.

Theorem 11. Let Banach spaces $\mathcal{X}, \mathcal{Y}$ be reflexive, $(L, M) \in \mathcal{H}_{\alpha}\left(\theta_{0}, a_{0}\right), L_{1}^{-1} \in \mathcal{L}\left(\mathcal{Y}^{1} ; \mathcal{X}^{1}\right)$, for all $T>0$ $B \in C_{Q}^{\gamma(T)}([0, T] ; \mathcal{L}(\mathcal{U} ; \mathcal{Y})), g \in C_{Q}^{\gamma(T)}([0, T] ; \mathcal{Y}), \gamma(T) \in(0,1]$. Then system (18) is approximately controllable in free time, if and only if $\overline{\operatorname{span}}\left\{\operatorname{im}_{0}^{-1}(I-Q) B(T): T \in \mathbb{R}_{+}\right\}=\mathcal{X}^{0}$,

$$
\overline{\operatorname{span}}\left\{\operatorname{im} Y_{\alpha-1}(T-s) Q B(s): 0<s<T, T \in \mathbb{R}_{+}\right\}=\mathcal{Y}^{1}
$$

\section{Application to an Initial-Boundary Value Problem}

We shall apply the obtained criterion to the control system, which is described by an initial-boundary value problem for a partial differential equation, not solvable with respect to the time fractional derivative.

Let $\alpha \in(1,2), a_{k} \in C([0, T] ; \mathbb{R}), k \in \mathbb{N}$, $\sup _{k \in \mathbb{N}}\left|a_{k}(t)\right|<\infty$ for every $t \in[0, T], v_{0}, v_{1} \in H_{0}^{2}(0, \pi):=$ $\left\{x \in H^{2}(0, \pi): x(0)=x(\pi)=0\right\}$. Consider the initial-boundary value problem

$$
\begin{gathered}
v(0, t)=v(\pi, t)=v_{\xi \xi}(0, t)=v_{\xi \xi}(\pi, t)=0, \quad t \in(0, T], \\
v(\xi, 0)+v_{\xi \xi}(\xi, 0)=v_{0}(\xi), \quad \xi \in(0, \pi), \\
v_{t}(\xi, 0)+v_{\xi \xi t}(\xi, 0)=v_{1}(\xi), \quad \xi \in(0, \pi),
\end{gathered}
$$

to the equation

$$
D_{t}^{\alpha}\left(v+v_{\xi \xi}\right)=v_{\xi \xi}+2 v_{\xi \xi \xi \xi}+\sum_{k=1}^{\infty} a_{k}(t)\langle u(\eta, t), \sin k \eta\rangle_{L_{2}(0, \pi)} \sin k \xi, \quad(\xi, t) \in(0, \pi) \times(0, T] .
$$

Choose $\mathcal{X}=H_{0}^{2}(0, \pi), \mathcal{Y}=\mathcal{U}=L_{2}(0, \pi), L=1+\frac{\partial^{2}}{\partial \xi^{2}} \in \mathcal{L}(\mathcal{X} ; \mathcal{Y})$,

$$
\begin{gathered}
\mathcal{D}_{M}=H_{0}^{4}(0, \pi):=\left\{x \in H^{4}(0, \pi): x(0)=x(\pi)=x^{\prime \prime}(0)=x^{\prime \prime}(\pi)=0\right\} \\
M=\frac{\partial^{2}}{\partial \xi^{2}}+2 \frac{\partial^{4}}{\partial \xi^{4}} \in \mathcal{C} l(\mathcal{X} ; \mathcal{Y}), \quad B(t)=\sum_{k=1}^{\infty} a_{k}(t)\langle\cdot, \sin k \eta\rangle_{L_{2}(0, \pi)} \sin k \xi \in \mathcal{L}(\mathcal{U} ; \mathcal{Y}), t \in[0, T] .
\end{gathered}
$$

Thus, problem (25)-(28) has form (17) and (18) with $g \equiv 0$. Here we have ker $L=\operatorname{span}\{\sin \xi\} \neq$ $\{0\}$, hence Equation (28) is degenerate.

It is known that the set $\{\sqrt{2 / \pi} \sin k \xi: k \in \mathbb{N}\}$ is the orthonormal basis in $L_{2}(0, \pi)$ of eigenfunctions of the operator $\frac{\partial^{2}}{\partial \xi^{2}}$ with domain $H_{0}^{2}(0, \pi)$, which correspond to the eigenvalues $\left\{-k^{2}: k \in \mathbb{N}\right\}$. Since the polynomials $1+\lambda$ and $\lambda+2 \lambda^{2}$ have no common roots, by Theorem 7 [22] the operator $L_{1}: \mathcal{X}^{1} \rightarrow \mathcal{Y}^{1}$ is a homeomorphism and for $\alpha \in[1,2)$ there exist $\theta_{0} \in(\pi / 2, \pi), a_{0} \geq 0$, such that $(L, M) \in \mathcal{H}_{\alpha}\left(\theta_{0}, a_{0}\right)$. Besides, from Theorem 7 [22] it follows, that $\mathcal{X}^{0}=\mathcal{Y}^{0}=\operatorname{span}\{\sin \xi\}, \mathcal{Y}^{1}$ is the closure of $\operatorname{span}\{\sin k \xi: k=2,3, \ldots\}$ in $L_{2}(0, \pi), \mathcal{X}^{1}$ is the closure of the same set in $H_{0}^{2}(0, \pi)$.

By Lemma 3 subsystem (21) is controllable in time $T$, if and only if $a_{1}(T) \neq 0$. Besides, it is controllable in free time if and only if $a_{1} \not \equiv 0$ on $\mathbb{R}_{+}$. In the both cases we can say about the exact controllability on the one-dimensional space $\mathcal{X}^{0}$. 
For $y \in L_{2}(0, \pi)$ we have

$$
\begin{aligned}
Y_{\alpha-1}(t) y & =\sum_{k=2}^{\infty} y_{k} \sin k \xi \frac{1}{2 \pi i} \int_{\Gamma} \frac{e^{\mu t} d \mu}{\mu^{\alpha}-\mu_{k}}=\sum_{k=2}^{\infty} y_{k} \sin k \xi \frac{1}{2 \pi i} \int_{t \Gamma} \frac{t^{\alpha-1} e^{\lambda} d \lambda}{\lambda^{\alpha}-t^{\alpha} \mu_{k}}= \\
& =\sum_{k=2}^{\infty} y_{k} \sin k \xi \sum_{n=0}^{\infty} t^{\alpha(n+1)-1} \mu_{k}^{n} \frac{1}{2 \pi i} \int_{t \Gamma} e^{\lambda} \lambda^{-\alpha(n+1)} d \lambda= \\
= & \sum_{k=2}^{\infty} y_{k} \sin k \xi \sum_{n=0}^{\infty} \frac{t^{\alpha(n+1)-1} \mu_{k}^{n}}{\Gamma(\alpha(n+1))}=\sum_{k=2}^{\infty} t^{\alpha-1} E_{\alpha, \alpha}\left(\mu_{k} t^{\alpha}\right) y_{k} \sin k \xi
\end{aligned}
$$

where

$$
y_{k}=\langle y(\eta), \sin k \eta\rangle_{L_{2}(0, \pi)}, \quad \mu_{k}=\frac{2 k^{4}-k^{2}}{1-k^{2}}, \quad E_{\alpha, \beta}(z)=\sum_{n=0}^{\infty} \frac{z^{n}}{\Gamma(\alpha n+\beta)}
$$

is the Mittag-Leffler function. So,

$$
Y_{\alpha-1}(T-s) Q B(s)=\sum_{k=2}^{\infty} a_{k}(s)(T-s)^{\alpha-1} E_{\alpha, \alpha}\left(\mu_{k}(T-s)^{\alpha}\right)\langle\cdot, \sin k \eta\rangle_{L_{2}(0, \pi)} \sin k \xi,
$$

therefore, subsystem (20) is approximately controllable in time $T$, if and only if for every $k \in \mathbb{N} \backslash\{1\}$ there exists $s_{k} \in(0, T)$, such that

$$
a_{k}\left(s_{k}\right) E_{\alpha, \alpha}\left(\frac{2 k^{4}-k^{2}}{1-k^{2}}\left(T-s_{k}\right)^{\alpha}\right) \neq 0 .
$$

Since $E_{\alpha, \alpha}$ is the entire function and has isolated zeros only, such a condition is equivalent to the condition: $a_{k} \not \equiv 0$ on $[0, T]$ for every $k \in \mathbb{N} \backslash\{1\}$.

Analogously, subsystem (20) is approximately controllable in free time if and only if $a_{k} \not \equiv 0$ on $\mathbb{R}_{+}$ for all $k \in \mathbb{N} \backslash\{1\}$.

Moreover, it is easy to check that

$$
\sup _{k=2,3, \ldots} \frac{2 k^{4}-k^{2}}{1-k^{2}} \leq 0
$$

therefore, $(L, M) \in \mathcal{H}_{\alpha}\left(\theta_{0}, a_{0}\right)$ for some $\theta_{0} \in(\pi / 2, \pi), a_{0} \geq 0$ in the case $\alpha \in(0,1]$ (see Theorem 7 [22]). Hence we can study problem (25), (26) and (28) with $\alpha \in(0,1]$ analogously.

Proposition 1. Let $\alpha \in(0,2)$. System (25) and (28) is approximately controllable in time $T$ if and only if $a_{1}(T) \neq 0$ and for every $k \in \mathbb{N} \backslash\{1\} a_{k} \not \equiv 0$ on $[0, T]$.

Analogously, we can obtain the next assertion by the obvious way.

Proposition 2. Let $\alpha \in(0,2)$. System (25) and (28) is approximately controllable in free time, if and only if $a_{k} \not \equiv 0$ on $\mathbb{R}_{+}$for all $k \in \mathbb{N}$.

\section{Approximate Controllability of Systems with Finite-Dimensional Input}

Let $g:[0, T] \rightarrow \mathcal{Y}, b_{i} \in \mathcal{Y}, i=1,2, \ldots, n$, be given. Consider the control system

$$
D_{t}^{\alpha} L x(t)=M x(t)+\sum_{i=1}^{n} b_{i} u_{i}(t)+g(t)
$$


where $u_{i}:[0, T] \rightarrow \mathbb{R}, i=1,2, \ldots, n$. It is a partial case of system (18). Indeed, we can take $\mathcal{U}=\mathbb{R}^{n}, u=\left(u_{1}, u_{2}, \ldots, u_{n}\right), B u(t)=\sum_{i=1}^{n} b_{i} u_{i}(t)$. Such a control system is called a system with finite-dimensional input. It is evident that $B \in \mathcal{L}\left(\mathbb{R}^{n} ; \mathcal{Y}\right)$. Control function $u=\left(u_{1}, \ldots, u_{n}\right)$ will be chosen from the space $C^{\gamma}\left([0, T] ; \mathbb{R}^{n}\right)$. Theorem 10 and Theorem 11 implies the next assertion.

Corollary 1. Let Banach spaces $\mathcal{X}, \mathcal{Y}$ be reflexive, $(L, M) \in \mathcal{H}_{\alpha}\left(\theta_{0}, a_{0}\right), L_{1}^{-1} \in \mathcal{L}\left(\mathcal{Y}^{1} ; \mathcal{X}^{1}\right), b_{i} \in \mathcal{Y}$, $i=1,2, \ldots, n, g \in C_{Q}^{\gamma}([0, T] ; \mathcal{Y})$ for some $\gamma \in(0,1]$. Then

(i) system (29) is approximately controllable in time $T$ if and only if

$$
\operatorname{span}\left\{(I-Q) b_{i}, i=1,2, \ldots, n\right\}=\mathcal{Y}^{0}, \quad \overline{\operatorname{span}}\left\{Y_{\alpha-1}(s) Q b_{i}, 0<s<T, i=1,2, \ldots, n\right\}=\mathcal{Y}^{1} .
$$

(ii) system (29) is approximately controllable in free time if and only if

$$
\operatorname{span}\left\{(I-Q) b_{i}, i=1,2, \ldots, n\right\}=\mathcal{Y}^{0}, \quad \overline{\operatorname{span}}\left\{Y_{\alpha-1}(s) Q b_{i}, s \in \mathbb{R}_{+}, i=1,2, \ldots, n\right\}=\mathcal{Y}^{1} .
$$

Proof. By Theorem 10 the condition $\mathcal{X}^{0}=\overline{\operatorname{im}} M_{0}^{-1}(I-Q) B=\overline{\operatorname{span}}\left\{M_{0}^{-1}(I-Q) b_{i}, i=1,2, \ldots, n\right\}$ is necessary and sufficient for the approximate controllability in time $T$ of the subsystem on the subspace $\mathcal{X}^{0}$. This set is finite-dimensional, and the operator $M_{0}$ is densely defined, therefore

$$
\mathcal{X}^{0}=\operatorname{span}\left\{M_{0}^{-1}(I-Q) b_{i}, i=1,2, \ldots, n\right\}=\mathcal{D}_{M_{0}}
$$

it is equivalent to the equality $\mathcal{Y}^{0}=M\left[\mathcal{D}_{M_{0}}\right]=\operatorname{span}\left\{(I-Q) b_{i}, i=1,2, \ldots, n\right\}$. Other equalities follow from Theorems 10 and 11 in an obvious way.

Remark 13. So, we see that under the conditions of Corollary 1 from the approximate controllability of system (29) it follows that $\operatorname{dim} \mathcal{X}^{0}=\operatorname{dim} \mathcal{Y}^{0} \leq n$.

Remark 14. In the conditions of Corollary 1 from the approximate controllability of system (29) it follows that $M_{0} \in \mathcal{L}\left(\mathcal{X}^{0} ; \mathcal{Y}^{0}\right)$, since $\mathcal{D}_{M_{0}}=\mathcal{X}^{0}$ and the operator $M_{0}$ is closed.

Let $\triangle$ be the Laplace operator and the system with one-dimensional input be described by the equation

$$
D_{t}^{\alpha}(5 v+\triangle v)=\triangle v+2 \triangle^{2} v+b(\xi, \eta) u(t), \quad(\xi, \eta, t) \in(0, \pi) \times(0, \pi) \times(0, T],
$$

with initial conditions of form (26) at $\alpha \in(0,1]$, or of form (26), (27) at $\alpha \in(1,2)$, defined on $(0, \pi) \times(0, \pi)$ and with boundary conditions of the form

$$
\begin{gathered}
v(0, \eta, t)=v(\pi, \eta, t)=v(\xi, 0, t)=v(\xi, \pi, t)=0, \quad \xi, \eta \in(0, \pi), t \in(0, T], \\
\triangle v(0, \eta, t)=\triangle v(\pi, \eta, t)=\triangle v(\xi, 0, t)=\triangle v(\xi, \pi, t)=0, \quad \xi, \eta \in(0, \pi), t \in(0, T] .
\end{gathered}
$$

Here $b \in L_{2}((0, \pi) \times(0, \pi))$. Reasoning as in Section 6, we see that system (30)-(32) is not controllable in free time even, since the subspace $\mathcal{Y}^{0}=\operatorname{span}\{\sin \xi \sin 2 \eta, \sin 2 \xi \sin \eta\}$ is two-dimensional, and the condition span $\{(I-Q) b\}=\mathcal{Y}^{0}$ can not be satisfied.

\section{Conclusions}

Thus, the work obtained the necessary and sufficient conditions for approximate controllability for a class of degenerate fractional order evolution equations in terms of operators from the equation. The cases of infinite-dimensional and finite-dimensional input were studied. Using the concrete 
control systems described by the initial-boundary value problems for the partial differential equations, the applications of the obtained abstract results were demonstrated.

Author Contributions: Conceptualization, D.B.; methodology, D.B. and V.E.F.; validation, D.M.G. and K.T.; formal analysis, V.E.F., D.M.G. and K.T.; investigation, D.M.G. and K.T.; Writing-Original Draft preparation, V.E.F.; Writing-Review and Editing, D.B. and V.E.F.; supervision, D.B. and V.E.F.; project administration, V.E.F. and D.M.G.

Funding: The reported study was funded by the Russian Foundation for Basic Research, project number 19-41-450001; by Act 211 of Government of the Russian Federation, contract 02.A03.21.0011; and by Ministry of Science and Higher Education of the Russian Federation, task number 1.6462.2017/BCh.

Conflicts of Interest: The authors declare no conflict of interest.

\section{References}

1. Kalman, R.E.; Ho, Y.S.; Narendra, K.S. Controllability of linear dynamical systems. Contrib. Differ. Equ. 1963, 1, 189-213.

2. Krasovskii, N.N. On the theory of controllability and observability of linear dynamic systems. J. Appl. Math. Mech. 1964, 28, 1-14. [CrossRef]

3. Fattorini, H.O. On complete controllability of linear systems. J. Differ. Equ. 1967, 3, 391-402. [CrossRef]

4. Kurzhanskiy, A.B. Towards controllability in Banach spaces. Differ. Equ. 1969, 5, 1715-1718. (In Russian)

5. Triggiani, R. Controllability and observability in Banach space with bounded operators. SIAM J. Control 1975, 13, 462-491. [CrossRef]

6. Curtain, R.F. The Salamon-Weiss class of well-posed infinite dimensional linear systems: A survey. IMA J. Math. Control Inf. 1997, 14, 207-223. [CrossRef]

7. Sholokhovich, F.A. On controllability of linear dynamical systems. News Ural State Univ. 1998, 10, $103-126$. (In Russian)

8. Debbouche, A.; Baleanu, D. Controllability of fractional evolution nonlocal impulsive quasilinear delay integro-differential systems. Comput. Math. Appl. 2011, 62, 1442-1450. [CrossRef]

9. Chalishajar, D.N.; Malar, K.; Karthikeyan, K. Approximate controllability of abstract impulsive fractional neutral evolution equations with infinite delay in Banach spaces. Electron. J. Differ. Equ. 2013, 2013, 1-21.

10. Fedorov, V.E.; Ruzakova, O.A. Controllability of linear Sobolev type equations with relatively $p$-radial operators. Russ. Math. 2002, 46, 54-57.

11. Fedorov, V.E.; Ruzakova, O.A. Controllability in dimensions of one and two of Sobolev-type equations in Banach spaces. Math. Notes 2003, 74, 583-592. [CrossRef]

12. Fedorov, V.E.; Shklyar, B. Exact null controllability of degenerate evolution equations with scalar control. Sbornik Math. 2012, 203, 1817-1836. [CrossRef]

13. Plekhanova, M.V.; Fedorov, V.E. Optimal Control for Degenerate Distributed Systems; Publishing Center of South Ural State University: Chelyabinsk, Russia, 2013. (In Russian)

14. Plekhanova, M.V.; Fedorov, V.E. On controllability of degenerate distributed systems. Ufa Math. J. 2014, 6, 77-96. [CrossRef]

15. Fedorov, V.E.; Gordievskikh, D.M.; Baybulatova, G.D. Controllability of a class of weakly degenerate fractional order evolution equations. AIP Conf. Proc. 2017, 1907, 020009-1-020009-14.

16. Fedorov, V.E.; Gordievskikh, D.M.; Turov, M.M. Infinite-dimensional and finite-dimensional $\varepsilon$-controllability for a class of fractional order degenerate evolution equations. Chelyabinsk Phys. Math. J. 2018, 3, 5-26. (In Russian)

17. Fedorov, V.E.; Gordievskikh, D.M. Approximate controllability of strongly degenerate fractional order system of distributed control. In Proceedings of the 17th IFAC Workshop on Control Applications of Optimization CAO 2018, Yekaterinburg, Russia, 15-19 October 2018; Volume 51, pp. 675-680.

18. Plekhanova, M.V. Distributed control problems for a class of degenerate semilinear evolution equations. J. Comput. Appl. Math. 2017, 312, 39-46. [CrossRef]

19. Plekhanova, M.V. Optimal control for quasilinear degenerate distributed systems of higher order. J. Math. Sci. 2016, 19, 236-244. [CrossRef]

20. Bajlekova, E.G. Fractional Evolution Equations in Banach Spaces. Ph.D. Thesis, University Press Facilities, Eindhoven University of Technology, Eindhoven, The Netherlands, 2001. 
21. Prüss, J. Evolutionary Integral Equations and Applications; Springer: Basel, Switzerland, 1993.

22. Fedorov, V.E.; Romanova, E.A.; Debbouche, A. Analytic in a sector resolving families of operators for degenerate evolution fractional equations. J. Math. Sci. 2018, 228, 380-394. [CrossRef]

23. Fedorov, V.E. A class of fractional order semilinear evolutions in Banach spaces. In Integral Equations and Their Applications, Proceedings of the University Network Seminar on the Occasion of the Third Mongolia-Russia-Vietnam Workshop on NSIDE 2018, Hung Yen, Vietnam, 27-28 October 2018; Hanoi Mathematical Society, Hung Yen University of Technology and Education: Hung Yen, Vietnam, 2011; pp. 11-20.

24. Fedorov, V.E.; Romanova, E.A. Inhomogeneous evolution equations of fractional order in the sectorial case. Itogi Nauki i Tekhniki. Contemp. Math. and its Appl. Thematic Reviews. 2018, 149, 103-112. (In Russian)

25. Fedorov, V.E.; Gordievskikh, D.M.; Plekhanova, M.V. Equations in Banach spaces with a degenerate operator under a fractional derivative. Differ. Equ. 2015, 51, 1360-1368. [CrossRef]

(c) 2019 by the authors. Licensee MDPI, Basel, Switzerland. This article is an open access article distributed under the terms and conditions of the Creative Commons Attribution (CC BY) license (http://creativecommons.org/licenses/by/4.0/). 